\title{
An Integrated Behavioural Model towards Evaluating and Influencing Energy Behaviour-The Role of Motivation in Behaviour Demand Response
}

\author{
Julia Blanke *, Christian Beder and Martin Klepal \\ People Behaviour \& Technology Integration Group, Nimbus Research Centre, Cork Institute of Technology, \\ Cork T12P928, Ireland; christian.beder@cit.ie (C.B.); martin.klepal@cit.ie (M.K.) \\ * Correspondence: Julia.Blanke@cit.ie
}

Received: 22 September 2017; Accepted: 13 December 2017; Published: 16 December 2017

\begin{abstract}
The change in the actual use of buildings by its occupants is receiving more and more attention. Over the lifecycle of a building the occupants and therefore the demands towards the buildings often change a lot. To match these altering conditions, particularly in the context of the demand for energy efficiency, purely technical approaches usually cannot solve the problem on their own or are not financially viable. It is therefore essential to take the behaviour of the end user into account and ask the fundamental question: "How is it possible to influence people's behaviour towards a more pro-environmental outcome, and also in the long-term?" To approach this question we will present a model-driven approach for dynamically involving building occupants into the energy optimisation process. To do so we will further develop an integrated behavioural model based on established behavioural theories, having a closer look how motivational variables can be integrated into the process. This should lead to novel approaches for behaviour demand response, enabling additional demand shifting and shedding through targeted real-time engagement with energy prosumers.
\end{abstract}

Keywords: prosumer behaviour modelling; behaviour demand response; motivation; self-determination theory; action regulation theory; goal-setting theory; theory of planned behaviour

\section{Introduction}

The role of occupant behaviour in the energy consumption of modern buildings during their operational phase is becoming more and more important. In particular, the widening gap between actual energy consumption during this phase and the predicted energy consumption at the design stage requires measures for optimising the energy footprint of existing buildings by taking behavioural aspects into account much more than it has previously been done. In this paper we are going to present a modelling approach that allows describing individual energy end user's demand behaviour. It enables a rigorous model-driven energy optimisation process that is able to explicitly take the building occupant's behaviour into account.

Typical current energy systems assume a rather strict distinction between a consumer-driven demand-side behaviour on one end, and a matching supply side, usually provided by the energy grid, on the other end. The latter has the primary objective of always satisfying consumer demands regardless of the constraints on the supply side. Although energy suppliers have always modelled demand-side behaviour to adapt their production accordingly, and certain incentive schemes, like different tariffs, have always been in place to influence demand-side behaviour according to supply-side constraints, modern communication technology enables a much more granular and individualised approach. 
Furthermore, renewable energy sources are creating much more unpredictable, sometimes even unintuitive, supply-side constraints that would require very specific targeted actions on the demand side. At the same time the impact of such demand-side actions has become increasingly important due to improved insulation and energy efficiency in general. For instance, opening a window in the past did not have the same relative impact as insulation was poor anyway and the heat loss introduced by this behaviour was negligible in comparison to today's highly insulated and efficient buildings.

Moreover, local generation and storage facilities have led to a situation where the classical consumer also takes part in the supply side. The consumer being now able to provide this capability to the grid is effectively becoming what is called a prosumer these days.

To deal with this situation a couple of approaches have been taken: the easiest, but at the same time least cost-effective, is by maintaining sufficient overcapacity in both the grid, as well as energy production. Thus, the system is able to always meet any possible consumer demand by controlling production accordingly. In order to better utilise existing assets in this scenario, modelling of consumer behaviour and other external factors, most prominently the weather, has been a very common approach. However, supply-side constraints are never communicated to the consumer in this scenario, the energy grid is always completely invisible in this regard. More flexible energy tariffs combined with real-time information provided to the consumers have been proposed to overcome this issue; however, smart-meters have so far not shown the desired outcome in the long run [1,2].

The approach proposed in this paper aims at going one step further by presenting a behavioural model that allows not only modelling demand behaviour as an external factor to the system, but to incorporate the individual building occupant and his/her characteristics as an integral part into the system itself. By further developing the integrated behavioural theory presented in Blanke et al. [3] we will show how demand-side behaviour could be evaluated and influenced based on supply-side constraints to achieve better overall energy efficiency. Particular focus will be put on incorporating motivational factors into the proposed model.

The starting point of this approach are the fused theories of action regulation (ART) by Hacker [4], the high-performance cycle (HPC) by Locke and Latham [5], the theory of planned behaviour (TPB) by Ajzen [6], as well as the social cognitive theory (SCT) by Bandura [7]. Integrating those theories into each other a holistic cycle of behaviour is obtained [3], comprising of the basic aspects of human behaviour found in many other approaches. Through the fact that the "basic aspects of human behaviour" might be based on the perspective taken, the model tries to be sufficiently open, so that further suggestions can be taken into account and tested within the model. We will briefly outline these concepts in Section 2.1, followed by a description of the self-determination theory [8] in Section 2.2, which will be shown to be integrated into each other in Section 3. The latter, being the integration of motivational factors into the modelling of building occupant behaviour, is the major contribution of this paper.

We will show in the following how these theories can be adapted and augmented in order to facilitate a model driven energy optimisation framework. We will present a use-case, which shows how these models translated into a hidden Markov random field can be used to fuse various inputs data sources, generate custom real-time message towards the building occupants, and estimate the aggregated expected impact these message have on the energy consumption. This approach allows taking into account not only the supply-side constraints and available production assets, but also considering the building occupant him/herself as a contributor to the optimisation process.

\section{Theoretical Foundation}

\subsection{An Integrated Behaviuoalr Model}

We will first present a brief introduction to the integrated behavioural model presented in [3], which we use as a basis for the integration of motivational factors in Section 3. In a behaviour demand response scenario, supply-side constraints, for instance, arising from capacity limitations 
of the production or distribution assets, are causing demands (see Figure 1, green) on the prosumer from the perspective of the energy optimisation system. It is these supply-side constraints, which are triggering the execution of the behaviour demand response cycle modelled in the integrated behaviour model. They result in specific demands imposed on the individual, which can be found as concepts both in the action regulation theory (ART), as well as in the high-performance cycle (HPC).

These constraints are usually created by the dynamic nature of the sources of energy that arise for various reasons. For example, renewable energy sources are highly dependent on the weather conditions. Or, combined heat and power plants (CHP) have a fixed ratio between electricity and heat produced, all of which do not necessarily meet the demand-side requirements per se. Our goal is, therefore, to take these external supply-side constraints into account and translate them into adapted behavioural responses by exploiting demand flexibility, for example, by suggesting better times for using appliances like the washing machine or dryer. Of course this always has to take into consideration the context and individual aspects, one of which being the individual's motivation, which we are specifically focusing on in Sections 2.2 and 3.

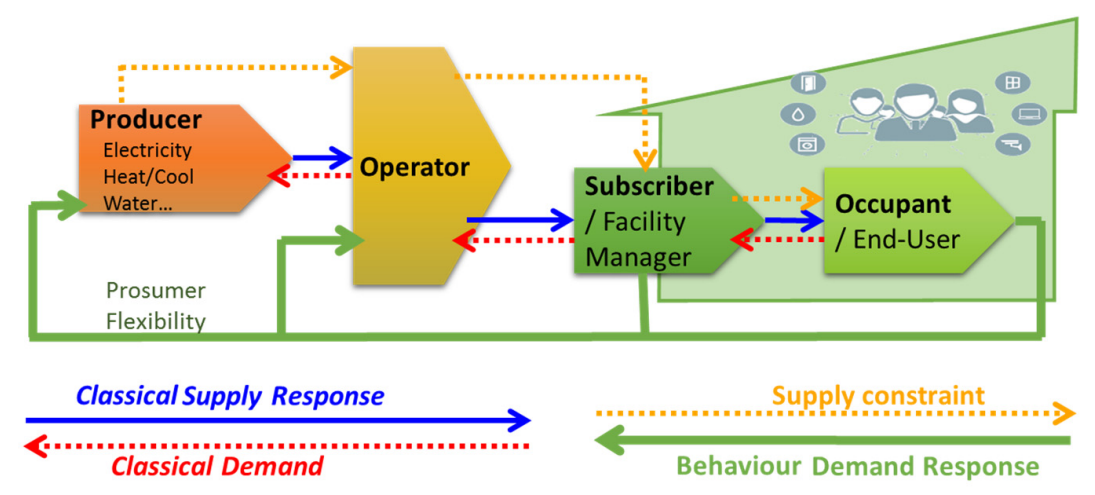

Figure 1. Additional value created by behaviour demand response in the energy supply chain.

In the HPC the action would follow immediately after these external demands, which are imposed onto the individual, leaving a big question mark about "What happens in between?" To clarify this question, the important aspect of redefinition of the task/suggestions are added from the ART [9] (Figure 2), stating that different people interpret the same task differently. The reason behind it is that every person interprets a demand on the base of individual motives, knowledge, experience, norms, attitudes, and personal traits. Furthermore the redefinition of task is a vehicle to personalise and simplify the underlying cognitive demands behind this process. That means a person should only choose those tasks, which in the context of the self-efficacy approach (social cognitive theory, [7]), " ... is what I believe I can do with my skills under certain conditions" [10], are achievable. Otherwise, the intention or probability that the behaviour in question is executed will decrease. Suggestions are influenced by norms, attitudes and control beliefs modelled in the theory of planned behaviour [6]. That gives us an indication about the intention that the behaviour in question will be executed by the individual. There is no point in suggesting goals which the prosumer is not intending to choose at all. Modelling all these variables explicitly even allows the energy optimisation process to go one step further and calculate an expected impact. Each suggested energy action will take by weighing them with the estimated intention to act for each individual. This allows the calculation of behaviour demand response flexibility, as well as enables the energy optimisation process to choose the right individual to interact with and trigger the necessary dynamic energy behaviour accordingly.

For example, an occupant of a smart building that is providing some level of interaction to its occupants for the purpose of energy optimisation should be receiving only information and suggestions relevant to him/her. Moreover, the smart building management system should be choosing the right individuals to perform specific tasks. This choice depends on its understanding of the individuals expected intention to act, as well as the expected impact such actions will have on the environmental 
variables. This only involves people into the optimisation process such that the specific current supply-side constraints are matched. This is in contrast to sending out unspecific and untargeted messages, which will very likely not be suitable to continuously adapt to the dynamically changing circumstances. According to the ART, personalised external demands and constrains resulting in overall personal goals and suggestions should be followed up by breaking them down into sub-goals with precise action plans (goal setting/anticipation; Figure 2). For example, the opening of windows, closing radiator valves, and so forth, are forming components of more complex chains of actions towards the set goals. The assumption is that such specific action plans have an amplifying effect on the intention to act. Furthermore, such consecutive action plans can be used to predict future demand profiles more precisely. For instance, in turning on the washing machine now, not only will the energy demand be shaped according to current supply-side constraints, but we will also be able to predict the demand profile arising from the next potential sub-goal of turning on the dryer afterwards more precisely. If, on the other hand, we do not detect the washing machine being turned on despite the suggestion to do so, we can then assume that the dryer will not run afterwards either. That is another demand profile prediction that can be explained by the feedback loops in the model. Detecting if specific actions are actually taken allows to constantly recalibrate the system and will make the estimation of the intention to act more reliable over time.

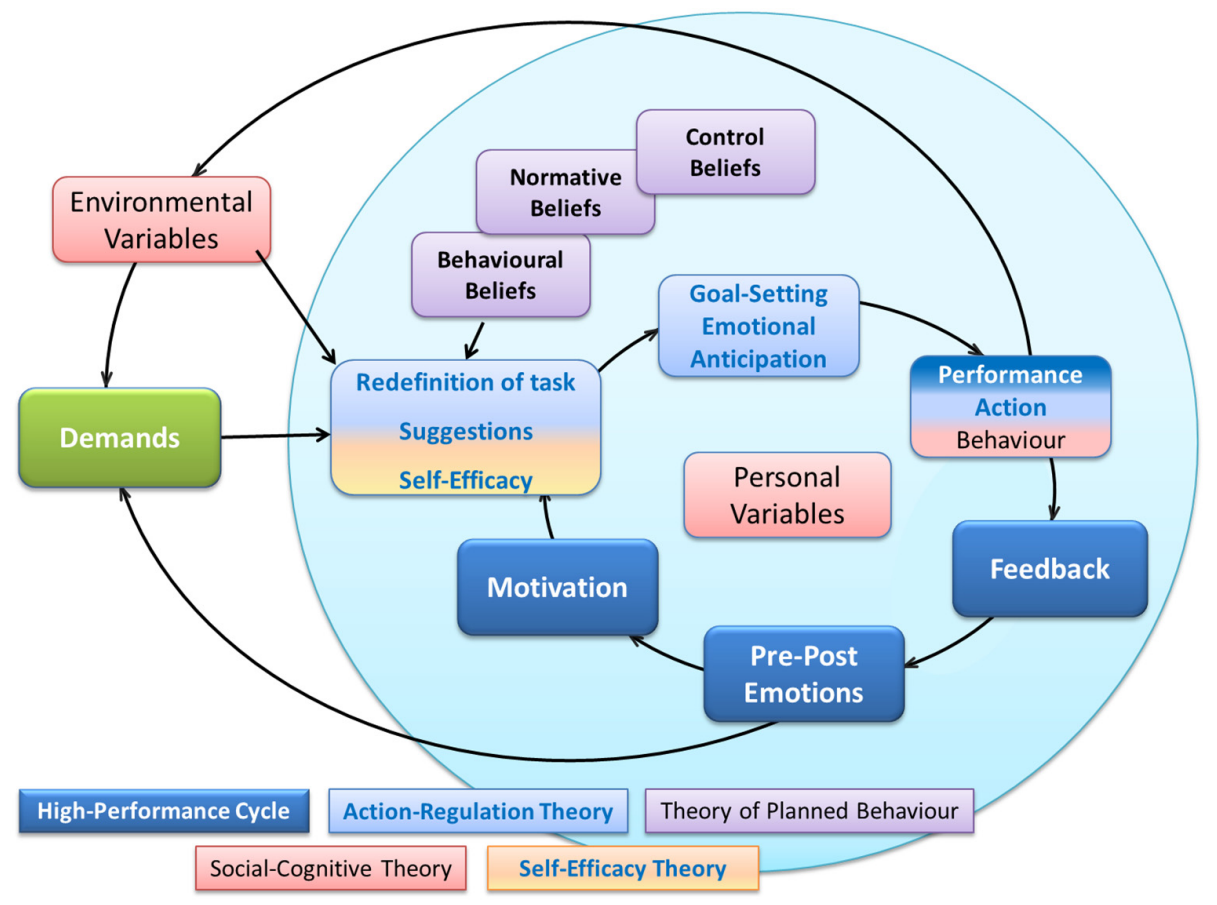

Figure 2. A holistic cycle of behaviour integrating aspects from different behavioural theories.

While in the underlying theories the performance results were seen to be the visible part of an action/behaviour in the context of industrial work, for example a product, in the context of energy behaviour performance results are much more complex to evaluate. Performance traditionally has been only measured from the outside at the end of an action through the visible results, where today there are much more flexible approaches via just-in-time analysis and feedback technologies. These new technologies are trying continuously to sense and monitor end-user behaviour, as well as the supply-side constraints, and are sending instant feedbacks to both sides to improve the interaction between both of them. However, this industrial example is also applicable to the energy domain. The performance results can be seen as matching supply-side constraints with adapted demand profiles. They are always monitored through sensors either as part of the classical building management systems (BMS), or through the ever increasing deployments of Internet of Things (IoT) sensors, which could be 
installed particularly for the purpose of enabling behaviour demand response scenarios such as the ones described here.

According to the social cognitive theory (SCT) [7], human behaviour never happens in isolation, but well embedded into an environmental context, and by acting/behaving this context gets influenced and changed, and this change influences back and changes the person who acted in this context. Performance results traditionally led to external as well as internal feedback, which influences well-being and level of contentment, which then strongly influences motivation. Motivation itself has a strong impact on how someone deals with demands again, finally closing the cycle [3,4]. As already mentioned, nowadays feedback is given on all levels in the behavioural cycle; always trying to keep the end user in the loop by constantly letting them know about their progress in achieving a set goal and at the same time increasing the motivation to continue.

So far we covered basic aspects of the cycle, starting with demands, redefinition of task, self-efficacy, attitudes, norms and control beliefs, goal setting and-emotional anticipation, performance and personal and environmental perspectives. Although feedback and emotions are key aspects for human behaviour, it is beyond the scope of this paper, and we intend to deliver a comprehensive elaboration of the aspects feedback and emotions in subsequent works. Feedback and emotions will be only discussed here as far as it is necessary to distinguish other concepts from it without claiming that this is already a full description. In the following we will be focusing on the aspect of motivation.

\subsection{The Self-Determination Theory}

In order to tackle the aspect of motivation in the behavioural cycle, we will be having a closer look at the self-determination theory in this section. The "self-determination theory (SDT) is an empirically based theory of human motivation, development, and wellness" [11]. It claims that there are three basic psychological needs, autonomy, competence, and relatedness, which are supported or thwarted by the social environment, hence leading to the strength of, as well as the type of motivation. Furthermore, the SDT comprises aspects like autonomous motivation, controlled motivation, and amotivation to predict the performance and the outcome of the same. Other aspects are concerned with the intrinsic and extrinsic motivation in relation to goals or aspirations. We will see in Section 3 how it relates to the energy domain, but will give a brief introduction to the theory here first.

The SDT describes a broad framework for the evaluation of human motivation and personality [8]. As an organismic dialectical approach it assumes that people are proactive, curious and growth oriented. The constant dialectic or interaction between individual and social context gives the opportunity to predict future psychological growth and well-being. These aspects are divided into six mini-theories of which the SDT comprises:

1. The cognitive evaluation theory is focusing on intrinsic motivation. Intrinsic motivation is defined as behaviour, which is enjoyable on its own. No incentives need to be given but the social environment can be crucial when it comes to support competence and autonomy, two aspects which are essential for nurturing intrinsic motivation [12].

2. The organismic integration theory is focusing on extrinsic motivation and its different traits. Extrinsic motivation is defined as behaviour, which is instrumental and externally incentivised. Extrinsic motivation can be described on a continuum from external regulation, introjection, and identification until integration being the closest to intrinsic motivation, or put more specifically: the more internalised a certain extrinsic behaviour/goal the more autonomous a person acts. Again, the social context plays a crucial role when it comes to enhance or forestall internalisation of values, goals or belief systems [13].

3. The causality orientations theory (COT) focuses on interpersonal differences and the dialectic involvement of each one with their environment. "COT describes and assesses three types of causality orientations: the autonomy orientation in which persons act out of interest in and valuing of what is occurring; the control orientation in which the focus is on rewards, gains, and 
approval; and the impersonal or amotivated orientation characterized by anxiety concerning competence" [13].

4. The basic psychological needs theory focuses on three innate psychological needs: autonomy, competence, and relatedness. If those needs are optimal met well-being will be the consequence while if these basic needs are thwarted ill-being can be predicted.

5. The goal contents theory focuses on intrinsic and extrinsic goals and their impact on motivation. Extrinsic goals like appearance are associated with less well-being, while intrinsic goals like caring for myself is associated with higher psychological wellness and growth.

6. The relationships motivation theory (RMT) is focusing on development and maintenance of close personal relationships [13]. The RMT claims that relationships are not optional but in general essential to gain well-being, while autonomy and competence should be supported from the same.

In the following section we will discuss all these aspects of the SDT, how they relate to energy behaviour in particular, and how they can be integrated into the overall cycle described in Section 2.1 for the purpose of modelling motivation in behaviour demand response scenarios.

\section{The Integration of the Self-Determination Theory to Incorporate Motivational Aspects}

In order to integrate the self-determination theory into the behavioural cycle outlined in Section 2.1, we will pick up on its six constituent mini-theories and discuss how they can be inserted to fill gaps and clarify aspects so far missing in the behavioural modelling used. In particular, we will do so when it comes to describing motivation and how it influences the redefinition of task following the demands imposed by the supply-side constraints in the behaviour demand response scenario outlined above.

\subsection{Extrinsic-Intrinsic Motivation Continuum}

The cognitive evaluation theory (CET) is focusing on intrinsic motivation being defined as behaviour, which is enjoyable on its own. In the energy behaviour demand response scenario this has to translate into an action that is intrinsically motivated, rather than driven by external incentives [13]. While it is difficult to think of a situation where energy saving based on this type of motivation is achieved, it is well conceivable that certain supply-side constraints require energy end users to consume more energy, for instance, to take the edge off production spikes. In that case energy consuming activities and actions might be suggested by the energy optimiser that align with the individual's interests and desires. It is important to note that for this type of motivation no incentives need to be given, however, the social environment can be crucial when it comes to support competence and autonomy, two aspects which are essential for nurturing intrinsic motivation [12]. In this sense it is a very desirable type of motivation from the perspective of the energy provider, as no or very little cost is involved. Therefore, optimising for achieving such type of motivation can be beneficial in many circumstances [13]. Some aspects of smart metering and/or social integration with regards to energy behaviour can be seen as aiming for this particular aspect of motivation.

Next is the organismic integration theory (OIT), which is focusing on extrinsic motivation and its different aspects. In contrast to the intrinsic motivation covered by the CET, extrinsic motivation is defined as behaviour, which is instrumental and externally incentivised. As such, this type of motivation has been the target of energy related incentive schemes for a long time [13], beginning with direct monetary rewards through different tariffs all the way to softer approaches like for instance competition-based feedback mechanism such as gamification. The OIT recognises this scale on which the extrinsic motivation exists, and describes it on a continuum from external regulation, introjection, and identification up until integration, the latter being the closest to intrinsic motivation. It is important to note that the more internalised a certain extrinsic behaviour/goal, the more autonomous a person acts. This means, again, that a person is both more likely to be motivated to act in the desired way, as well as easier/cheaper to motivate to do so, the closer the motivation is towards the intrinsic. Therefore 
also the type of extrinsic motivation is a cost optimisation goal for the energy supplier, with more cost-effective interventions achieved targeting people who are further towards the intrinsic on the type of motivation continuum. As with the CET, the social context plays a crucial role when it comes to enhance or forestall internalisation of values, goals or belief systems [13], which is instrumental for achieving the above optimisation goals.

To be more specific, externally set goals together with their respective incentives are followed depending on where on the extrinsic motivation continuum the energy prosumer is, accepting the goal more or less and reacting accordingly: the closer the prosumer is to the intrinsic motivation (Figure 3, right), the less feedback and incentive is required as the goal is more internalised and seen as a stronger personal interest. Vice versa, if the prosumer is externally regulated (Figure 3, left), a lot of feedback, incentives and/or punishments need to be given to attain the desired outcome.

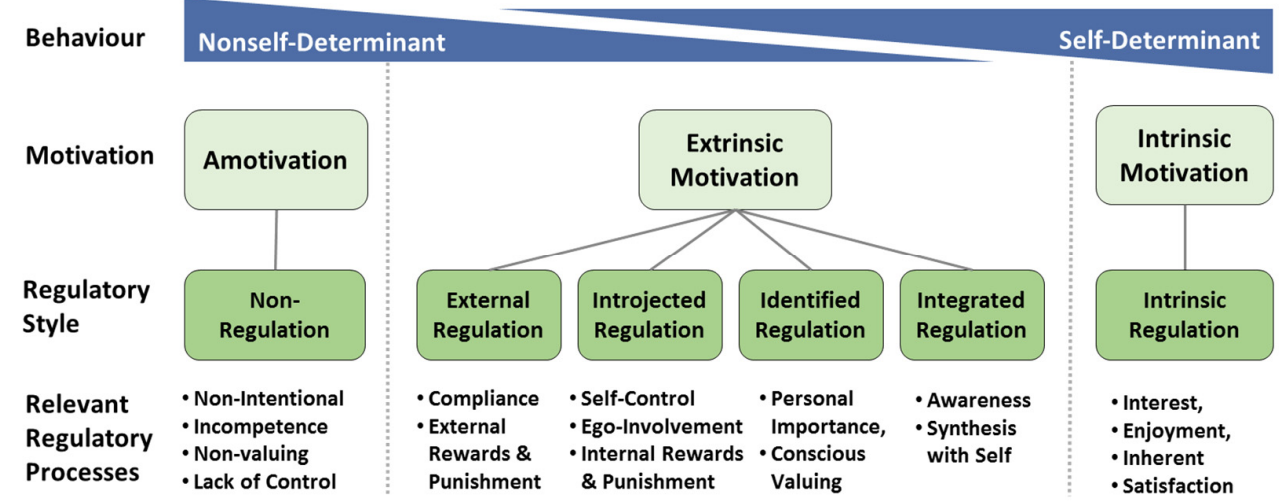

Figure 3. The self-determination continuum showing types of motivation with their regulatory styles, loci of causality, and corresponding processes [12].

The main drawback of the OIT is that the goal is externally set and not redefined as proposed in the action regulation theory (see above), where tasks need to be personalised. This could lead to potential prosumers not identifying with the goal and unnecessarily the overall motivation will be lower than it could be. Fusing the SDT with the cycle of behaviour outlined in Section 2.1 should overcome this shortcoming (Figure 2), with the motivation not directly linked to the action but going through a redefinition of task and goal setting stage first, as proposed by the ART (see Figure 4).

The goal contents theory (GCT) focuses on intrinsic and extrinsic goals and their impact on motivation. While the CET and OIT are concerned with the type of motivation, the GCT is more focused on the strength of motivation and its dependence on the goal contents. Extrinsic goals are associated with less well-being, while intrinsic goals are associated with higher psychological wellness and growth. The GCT shows high similarities to the redefinition of task/suggestions (Figure 1) as defined by the ART. The more personalised a task/goal is the higher the probability that the behaviour in question will be shown. The selection of the goal is defined by the type of motivation and the social context [11], hence the social context enhances or thwarts self-regulation and therefore influences the goal choice/internalisation. While the internalisation of goals is facilitated through the perception of autonomy, competence and context we can consider these aspects inside the self-efficacy concept, that is, " ... what I believe I can do with my skills under certain conditions" [10]. The higher the perceived autonomy and personal competence to show certain behaviour is the higher the possibility to attain the desired goal under the current circumstances. If, on the other hand, the control aspect is high in the environment, self-efficacy will decrease and with that the attainment of goals, too. 


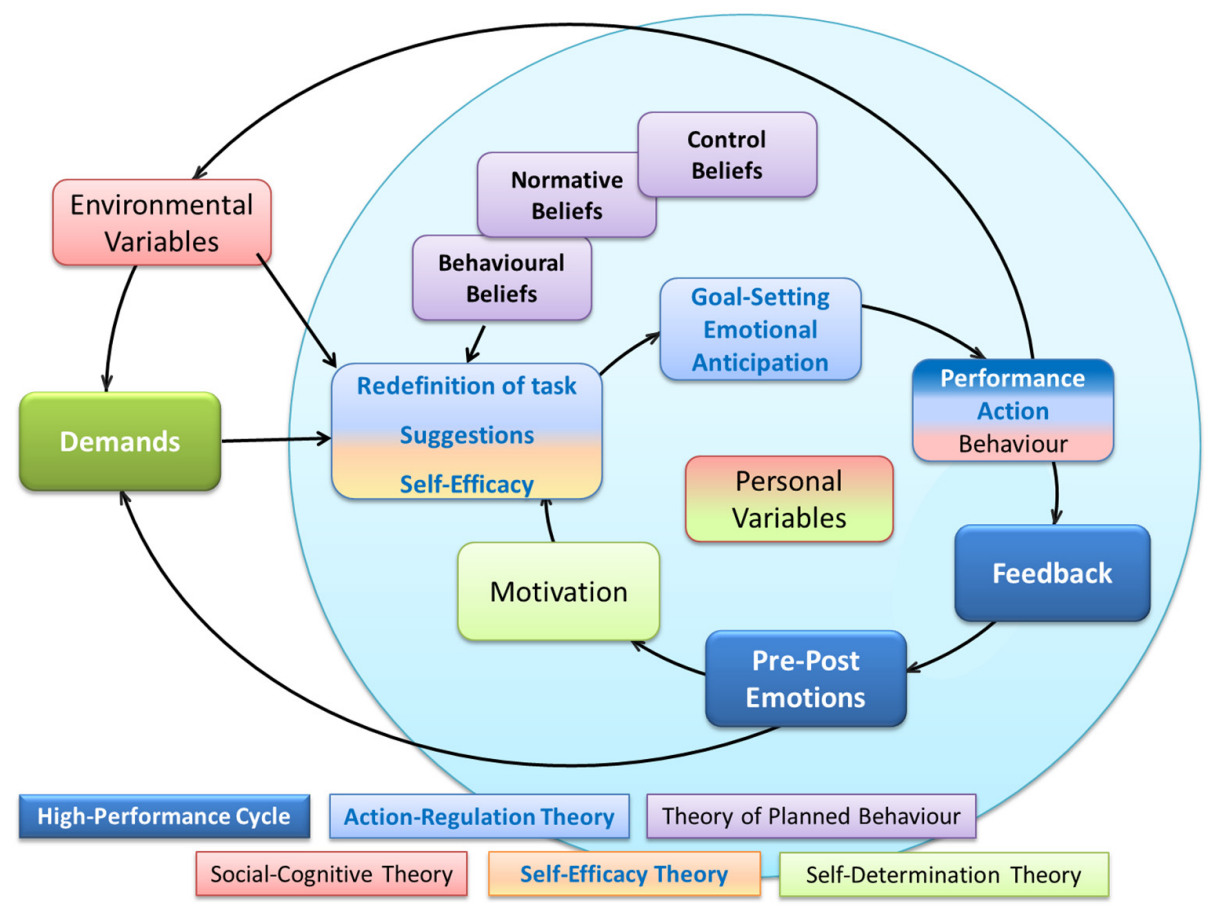

Figure 4. The self-determination theory integrated with the holistic behavioural cycle.

\subsection{Relation between Motivation and Intention}

Motivation is defined as an energizing process, a directing tendency and persistence [13]. It should not be confused with the "behavioural intention", which is defined here as the intention or the subjective probability of a person to perform various behaviours/the behaviour in question in respect to a given object [11]. In our context we see motivation more as a general tendency, while intention is the condition shortly before showing a behaviour in question. Short-term energy optimisation is focused on maximising the behavioural intention to act in order to make sure that the prosumer can be considered a reliable asset in the overall process. However, it is also important not to neglect the long-term motivation of building occupants. The strength of the motivation amplifies or dampens the intention to act and also the different types of motivation have different cost implications, as discussed above. Therefore, both should be considered important optimisation goals for the energy optimisation, as well beyond the short-term impact the optimisation of the intention to act can make.

In order to achieve this optimisation goal we observe that the extrinsic-intrinsic motivation continuum (Figure 3) provides information about the type of motivation a prosumer has. The intention provides information about the probability that a behaviour in question will be executed. By using evaluation methods from the SDT we know where the prosumer is on the continuum, which gives rise to the concept of potential of motivation (Figure 5). With that concept we can define the amplifying or dampening effect on the redefinition of task. Furthermore, we define the potential of intention coming from the accumulated data of the TPB (attitudes, norms and control beliefs), which has the same effect on the redefinition of task. Both quantities together are combined into the overall intention to act for a particular redefined goal. As outlined before, that is the most important short-term quantity to optimise for when it comes to exploiting behavioural demand flexibility. All three quantities together (type of motivation, potential of motivation, potential of intention) provide us with enough information to guide the suggestions given to the prosumer arising from different supply-side constraints in order to yield optimal results. Not only enables this information selecting the right individual, but also it allows to select the right tools and incentives to achieve the goals. For example, someone being externally regulated with regards to certain constraints needs strong incentives and feedback during the whole execution process, whereas someone more intrinsically driven will not, and the interaction with the 
prosumer should take this into consideration. In addition to this, adequate information, as well as awareness, can move the potential prosumer closer to the intrinsic motivation. Supporting autonomy and competence with clear action plans and giving the feeling of being part of something bigger will support this. It can also be supported by not just giving plain information, but embedding the whole system for instance into a gamification context depending on the personal interest of the end users.

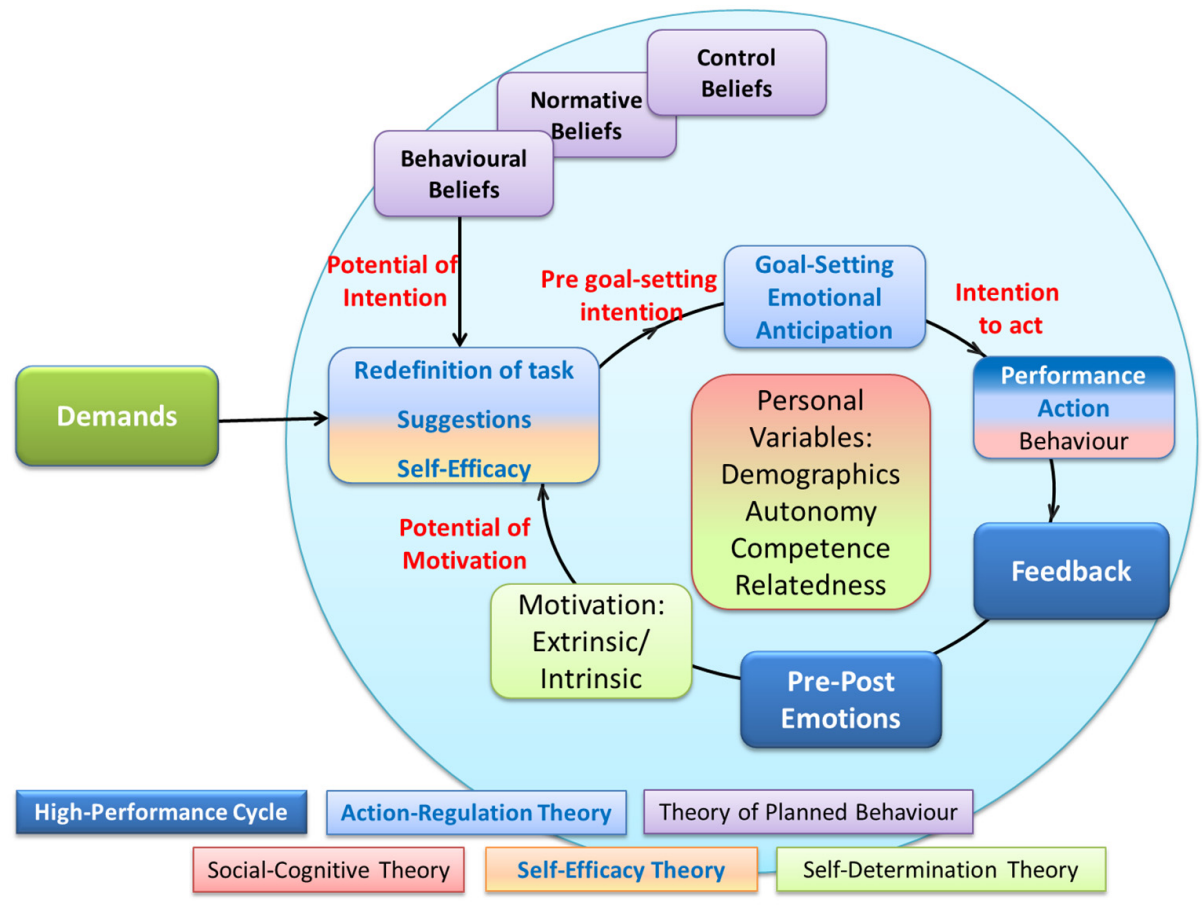

Figure 5. The potential of motivation and potential of intention as input parameters for the redefinition of task resulting in the pre-goal setting intention, increased by the goal setting, leading to the final intention to act.

\subsection{The Concept of Amotivation}

The causality orientations theory (COT) focuses on interpersonal differences and the dialectic involvement of each one with their environment. "COT describes and assesses three types of causality orientations: the autonomy orientation in which persons act out of interest in and valuing of what is occurring; the control orientation in which the focus is on rewards, gains, and approval; and the impersonal or amotivated orientation characterized by anxiety concerning competence" [13]. While the autonomy orientation is very similar to the concept of intrinsic motivation outlined above and the control orientation is related to the extrinsic motivation, the novel concept not covered before is amotivation. It describes a condition where no intention to act at all exists or even counterproductive behaviour appears [13]. "Amotivation results from a person not valuing a behaviour or outcome, not believing that a valued outcome is reliably linked to specific behaviours, or believing that there are behaviours instrumental to a valued outcome but not feeling competent to do those instrumental behaviours" [12]. This means that no matter what kind of incentives are given, the potential prosumer would not respond in a desired or expected way. The redefinition of task becomes an essential vehicle to help potential end users to overcome their disinterest. However, any energy optimisation process needs to be careful as to include into its schemes people who exhibit such counterproductive behaviour.

\subsection{Micro-Theories Supporting Other Sub-Concepts of the Behavioural Cycle}

The basic psychological needs theory (BPNT) focuses on three innate psychological needs: autonomy, competence, and relatedness. For example, autonomy in our model can be understood as 
the freedom to choose the goals independently, which for instance implies that a prosumer has to be competent to execute the chosen goals and feels as part of a bigger whole. If this prosumer would instead be externally controlled and had no freedom to choose or he/she would not be able to execute the tasks asked of him/her, motivation would drop significantly and instantly [13]. Therefore every effort has to be made to keep people in control of their own environment, if long-term commitment of the prosumer is required. The same is true if someone does not feel connected or related to the overall goal. Hence autonomy, competence and relatedness are vital to motivate people to contribute to the overall aims.

If these basic needs are optimally met, well-being will be the consequence, while if these basic needs are thwarted ill-being can be predicted. The basic needs can be seen as part of a general personality and, therefore, we will consider these aspects as personal variables in the cycle (Figure 5), rather than only considering them as part of the motivational block. As such, they will still affect motivation, but will be directly and explicitly linked to other concepts as well. Autonomy can be seen as part of the control beliefs of the TPB, as well as part of the self-efficacy concept. All three concepts are based on the perception and evaluation of the environment, if certain actions are possible to execute or not. Further to that the innate psychological needs can be used to define personal traits more precisely. The BPNT therefore enhances the concept previously considered as part of the personal variables by aspects of growth, personal development, well-being and curiosity. A detailed discussion of process- and content oriented motivation theories is beyond the scope of this paper.

Finally, the relationships motivation theory (RMT) is focusing on the development and maintenance of close personal relationships [8] and the impact this has on motivation. The RMT claims that relationships are not optional, but in general essential to gain well-being, while autonomy and competence should be supported from the same. As already mentioned above in the context of relatedness, relationships are influenced by the social context, as described in the SCT [7], with the social context influencing the individual and his/her relations to others and the environment, as well as the person influencing back towards the social context. It is obvious that actions only get a meaning in a social setting, and some approaches are trying to integrate social media and networks into energy optimisation processes in order to increase motivation. So far we have not considered social interactions in our behavioural model; however, we recognise the importance and intend to develop it into this direction in the future.

\subsection{Discussion}

Motivation as a whole is triggered through the anticipation of the goal, as well as the anticipation of intrinsic and extrinsic feedbacks. Furthermore, in terms of achieving or not achieving of a set goal, as well as getting or not getting the anticipated feedback and/or incentives influences motivation, there is no clear line where the cycle starts and ends. Motivation is always built by past experiences (anticipation) and the perception of the inter- and intrapersonal environment [7]. While the SDT defines the extrinsic motivation on a continuum from controlled to autonomous behaviour, Bandura describes the same phenomena via three different types of environment: imposed environment, selected environment and constructed environment [7], which more or less reflects the continuum of the SDT. Therefore the extrinsic motivation makes it more explicit how the environment influences motivation and behaviour than previously being considered in the behavioural model outlined in Section 2.1.

Personalisation or redefinition of task, which is one of the key concepts of the ART [9], is mentioned indirectly in the SDT on the continuum from extrinsic motivation to intrinsic motivation. The different stages of extrinsic motivation (external regulation, introjected regulation, identified regulation, integrated regulation and intrinsic regulation) can be understood as growing aspiration and identification with the set goal. It needs to be kept in mind that the same set goal is interpreted differently by each individual [4], which is why this process of redefinition is so crucial. In this aspect the SDT shows a gap when it comes to actual action plans and actions themselves, so that the integration into the cycle can offer a supplement to enhance the SDT with the missing aspects. 
As mentioned before, the aspect of redefinition is essential to increase the intention to act and show the behaviour in question [3]. The SDT states that controlled circumstances or goals need to be individualised to increase the personal involvement as laid out in the ART [9] as well. While the SDT is missing the regulation aspects emphasised in the ART, the ART, and with it the integrated cycle, is lacking when it comes to the motivational aspects. Integrating both, the process of redefinition of tasks or goals can be improved by considering the continuum from extrinsic to intrinsic motivation, if aspects of the TPB [3] and self-efficacy, as well as personal traits, are taken into account. We propose to introduce potential of motivation and potential of intention to account for both and integrate them into a joint intention to act, which is the posterior probability of taking an action. Suggestions for goal selection should be made taking into consideration the type of motivation, as provided by the SDT. As soon as adequate suggestions or goals are chosen, action plans can be created in order to maximise the final intention to show the behaviour in question, which is required by the given constraints, for instance, of the energy supplier.

\section{Case Study and Methodology}

The presented model describes a set of variables contained within each behavioural theory and their mutual relations. Furthermore, each of the presented theories provides validated inventories for assessing a subset of these input parameters. This leads to the graph structure indicated in Figure 4 being translated into a hidden Markov random field (HMRF), which describes each individual building occupant's behavioural profile in terms of a joint probability density function and its temporal evolution. Note that the picture in Figure 4 is only intended as a high-level overview, with each individual theory providing a set of relevant parameters to the HMRF. The second major contribution of the behavioural theories to the HMRF are the validated inventories, which present a means of observing partial states of the HMRF and therefore can be used to continuously calibrate the joint probability density function of variables. Finally, the third contribution to the estimation of building occupants' behavioural profiles represented by the HMRF is the link between action and environmental variables (see Figure 4), which can be measured in the physical environment by means of Internet of Things (IoT) devices. While the assessment via questionnaires provides valuable initial calibration of the system, the continuous monitoring of the effect of actions on the environment can be achieved much more unobtrusive. Both, however, help to reduce the entropy of the HMRF, thereby allowing to improve the interaction between the building occupant and the energy system by having more accurate information available.

For instance, selecting individuals with an expected high intention to act (see Figure 5) enables the energy optimisation process to use interactions with this individual as a reliable and predictable asset in the process. As outlined above, variables relating to type of motivation (Figure 3), attitudes, goal setting, and so forth, are an important precursor variable for the final intention to act, which means that taking those into consideration when creating the interaction is expected to maximise the desired outcome. All of this enables an optimisation process, continuously evaluating the reaction of the building occupants to the messages sent. The optimisation goes both ways: model parameters are learned and refined based on the different reactions to different messages sent to the building occupant, essentially constituting an ongoing experiment where the optimiser is adjusting the behavioural parameters to the actual observed reactions. On the other hand, messages are adapted to the parameters learned, thereby maximising the expected outcome of the interaction between the system and the end users, enabling the effective use of individual building occupants as reliable assets in the optimisation process.

In order for this approach to be applicable, a means of communication with individual building occupants, as well as an IoT deployment for unobtrusive measuring, the effect of actions needs to be in place. To that end we created a behavioural testbed on the Cork Institute of Technology (CIT) campus comprising:

a campus smartphone app, which has been augmented with components for sending real-time push notifications and means for dynamic continuous assessments via in-app surveys; 
- $\quad$ an indoor localisation system, enabling the precise contextualisation of sent messages taking into consideration the individual occupants' location within the building;

- a connection to the campus building management systems (BMS), enabling to react to dynamic requirements of the building heating systems, as well as collecting information from all wired sensors;

- a low-power long-range (LoRa)-based IoT deployment, enabling the collection of measurements relating to occupants' actions.

The experiment covers the whole of the CIT campus using three LoRa gateways (see Figure 6). In total 21 rooms have been equipped with 250 wireless sensors across almost all heating zones (see Figure 7). Sensors are measuring the operation of 31 openable windows, 27 radiators, and 11 additional electric heaters (see Figure 8). Also, occupancy, air quality, and room temperature are measured. The rooms comprise 11 offices, 8 classrooms, and two labs. A total of 18 participants were recruited to evaluate the extended version of the mobile application (see Figure 9).

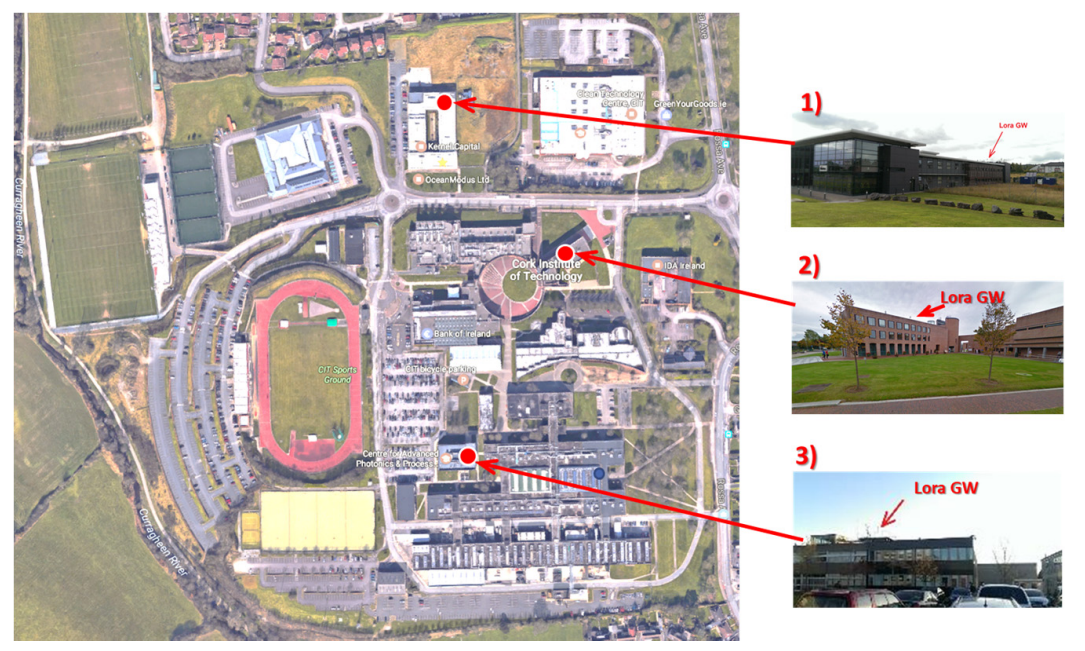

Figure 6. The low-power long-range (LoRa) network on the Cork Institute of Technology (CIT) campus: three gateways were installed in order to cover the whole campus, indoor and outdoor.
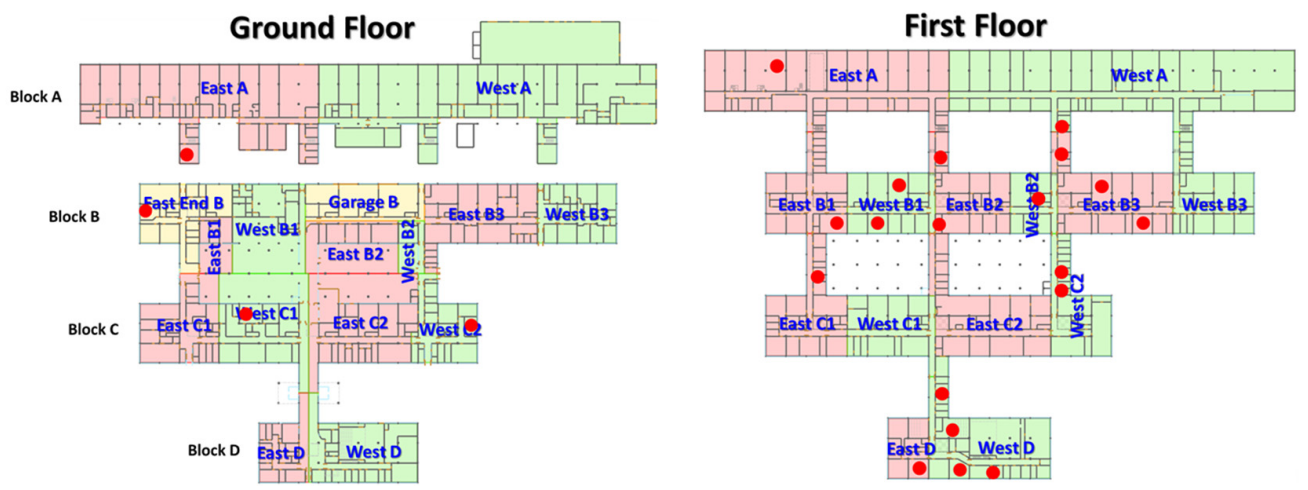

Figure 7. The placement of LoRa sensors across the different heating zones. 


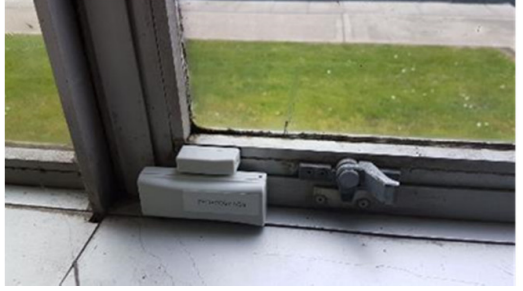

(a)

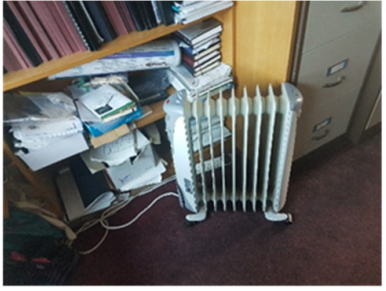

(b)

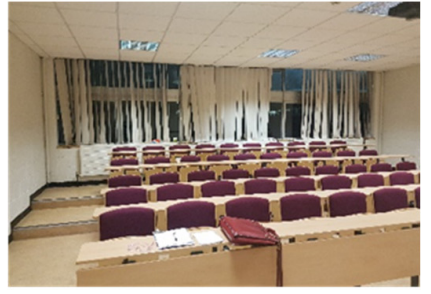

(c)

Figure 8. Placement of LoRa sensors. (a) Window open/close sensor. (b) Electric radiator temperature sensor in an office. (c) Radiator temperature sensor/passive infrared (PIR) occupancy sensor in a classroom.

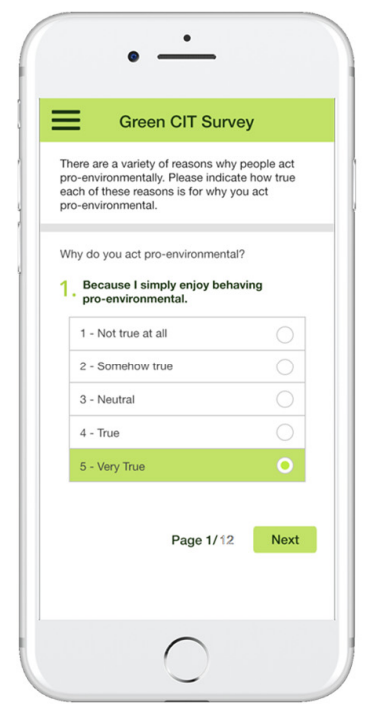

(a)

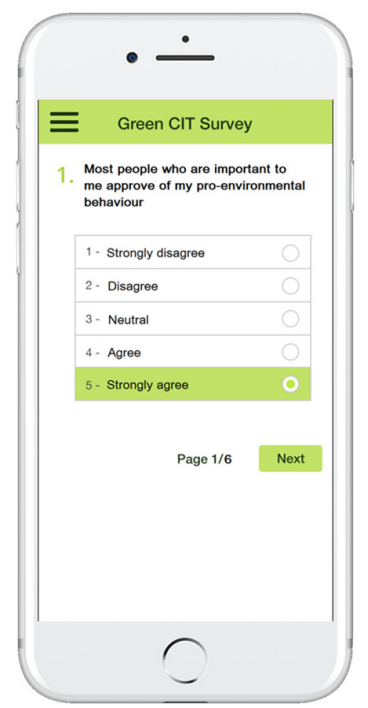

(b)

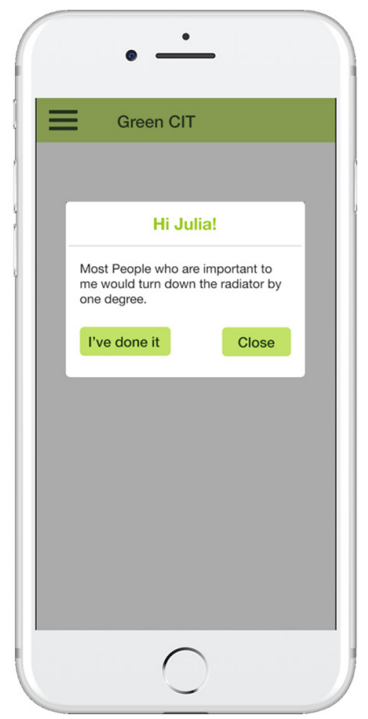

(c)

Figure 9. Screenshots of the phone application. (a) An example for the motivational self-regulation questionnaire proposed by the self-determination theory. (b) An example of a normative question as proposed by the theory of planned behaviour. (c) An example normative suggestion as generated by the energy optimiser.

The mobile application is integrated within the campus wide app, and provides some extra functionality for the purpose of behaviour demand response (BDR) management. This extra functionality is twofold: it enables triggering dynamic questionnaires based on the inventories proposed by the behavioural theories outlined above for the purpose of calibrating the HMRF accordingly (see Figure 9a). Secondly, it provides the energy optimisation system with the ability to send tailored push-notifications to individual occupants. This enables the implementation of BDR for individual heating zones asking participants to perform certain behaviours. Taking into account the supply-side demands for a specific heating zone as reported by the main building BMS, the context of the individual as measured by the indoor localisation system, the estimated parameters from the behavioural model as calibrated before, specific messages are sent to the individual maximising his/her intention to act on the suggestion as calculated from the model (see Figure 9c).

The main hypothesis to be evaluated is how the presented model-driven behavioural approach influences energy behaviour also in the long term. To approach this we have a number of sub-hypotheses:

1. The intention to act can be amplified by supporting the redefinition of the task, as well as the goal setting and goal anticipation aspects. 
2. By assessing the motivational type (intrinsic or extrinsic motivated) it is possible to identify the right actions and feedback which need to be executed to maintain long-term motivation with regard to the intention to act.

3. By knowing the intention to act of the individual it is possible to calculate the expected impact on the environment [14] and a better communication and adaptation to the supply-side constraints is possible.

4. By assessing motivational and intentional variables, we do not rely on less relevant statistics, for example, demographics, to make individual suggestions for the right person.

To test the hypothesis the HMRF needs to be calibrated first. This is done initially through questionnaires and, as the system is running, through the interaction of the participants with the application, as well as the sensed environment. In the first step the potential of intention, as well as the potential of motivation (see Figures 5 and 9) is assessed through questionnaires based on inventories proposed and validated in the TPB and SDT, respectively. Applying validated inventories to assess these precursor variables is one of the key advantages of the model-driven approach presented here, enabling the accurate measurement of behaviour relevant parameters taking advantage of established state-of-the art approaches [8]. The questionnaires, or part thereof, are delivered through the smartphone app already taking the answers into account when guiding through the questions. The specific information about behavioural beliefs, normative beliefs and control beliefs, as defined by the TPB and the intrinsic, and extrinsic motivation, as defined by the SDT, are assessed first, providing explicit information about the way how the system needs to communicate with the participant in the redefinition of the task. The redefinition of task is the process of personalising the supply-side constraints taking into account the current best estimate of these variables by the HMRF. Specifically, the TPB provides us with information about the way how the suggestions need to be phrased (sub-hypothesis 1, part 1). That means, for example, a person who shows strong normative beliefs needs suggestions like "Most people like me would turn down the radiator by one degree now." In addition to this, the motivation gives primarily information about the feedback type, if incentives are needed or if a certain type of information is requested [15] (sub-hypothesis 2).

The next step is not just telling people what to do, but how to do it. That seems straightforward for turning on a radiator or opening a window, but it can be more complex, for instance, when handling thermostat or smart meter settings. Clear action plans and guidance needs to be given, particularly to those who are afraid of using such appliances (sub-hypothesis 1, part 2). That means the application itself needs to be intuitive, helpful and easy to use. After the action plans are formed (goal setting/goal anticipation, see Figure 4) the actual behaviour/action follows. Not only is the end-performance evaluated, but also all the sub-goals and the process towards the goal can be directly assessed through the IoT sensor deployment in real time. This continuous assessment of actual actions taken in reaction to the suggestions sent to the individual enables to refine these interactions accordingly. As part of the overall optimisation process to maximise the individuals' intention to act the optimal strategy will eventually be selected. It is based on the continuously updated and recalibrated HMRF to give just-in-time feedback, keeping the prosumer always in the loop.

Each piece of feedback regarding an action influences motivation, and motivation should influence the type of interaction as outlined above. By knowing which type of motivation a person has, we can make assumptions about the feedback this person needs, always taking into account that feedback needs to be personalised and contextualised $[16,17]$. For example, someone who is extrinsically motivated needs to be incentivised and, depending where on the continuum (see Figure 3) the person is, the type of incentive needs to be adjusted accordingly (sub-hypothesis 2). On the other end of the spectrum a prosumer who is intrinsically motivated needs little feedback or incentives because he/she enjoys the activity on its own. Intrinsic motivation can be supported by giving feedback, which supports the aspects autonomy and competence $[13,15]$, all ultimately leading to improved potential of motivation (see Figure 5). Together with the potential of intention the potential of motivation is positively correlated with the intention to act (sub-hypothesis 3 ). The intention to act 
is the probability that a participant is acting on the suggestions sent through the phone application. Together with the impact these actions have on the environment (e.g., the reduction in heating load created by closing the window), we can calculate the expected impact on the environment. This quantity is the impact weighed by the probability of its occurrence for each dynamic suggestion. This makes it possible to quantify the aggregated behavioural flexibility (e.g., the amount of energy available to be displaced by sending out messages) offered by this approach to the energy optimisation (sub-hypothesis 3), enabling the implementation of BDR using the behavioural flexibility as a capacitive asset. An optimised BDR system is aiming to send out the right messages to the right person at the right time in order to adapt to dynamic supply-side constraints arising from fluctuations in energy availability. The proposed approach enables to optimise this based on relevant variables, maximising the overall intention to act as the key driver for the adoption and long-term adherence of the system (sub-hypothesis 4).

The experimental setup enables the measurement of energy consumption in the sub-metered respective heating zones. Further to that the mobile application and the IoT sensor deployment allow measuring if the participants react to the suggestions sent to them. The latter is explicitly used to recalibrate the HMRF, therefore, the variables and resulting suggestions dynamically adapt. In this sense the approach is an ongoing social experiment, constantly adapting the interactions according to the potentially shifting outcomes, which is the key driver when it comes to the claim of long-term adherence. The sensors provide the ability to directly measure the actions taken in reaction to the suggestions sent, from which we can deduce the intention to act for each individual. This, in combination with the pre-calibration from the questionnaires, makes it possible to refine the model parameters while it is applied. The HMRF represents a joint probability density function of all behaviour relevant parameters for each individual. A qualitative evaluation of the use-case outlined above indicates that participants are driven towards more pro-environmental behaviour in the CIT behavioural testbed, indicating that the suggested approach can be made viable. In this sense the qualitative analysis of the monitored interactions supports the sub-hypothesises stated above. However, currently, only volunteers are participating, suggesting that a strong selection bias is present in the experiment. BDR studies carried out on larger scales [18] suggest statistical significant quantitative effects to be measurable using only a subset of the variables discussed above. A fully integrated approach should, therefore, lead to more refined results, and we intend to extend our testbed beyond its current scope in order to evaluate this.

\section{Conclusions}

Behavioural modelling is a useful tool when it comes to developing energy optimisation strategies that not only take physical assets into account, but also integrate the building occupants themselves into the process. It allows to take a more rigorous and systematic approach towards energy prosumer engagement not relying on ad hoc assumptions and ideas, but modelling relevant parameters and their relation instead. While some approaches, such as, for instance, smart metering or gamification, which have been tried in the past, can be considered to tackle some of the aspects described in here, a holistic model of behaviour trying to take into account all relevant aspects has the potential of being applicable to a wider range of people.

In this paper we focused on motivation in particular, and explored how the six mini-theories of the self-determination theory can be integrated into the overall behavioural model introduced in [3]. In summary we can make the following conclusions:

- $\quad$ The SDT provides us with a typology of approaches to increase the motivation. As such it allows to categorise prosumer engagement products and to formalise which motivational parameters are targeted by these.

- The type of motivation can be seen as a guiding principle for the design of the intervention. It allows to design customised suggestions and tasks to influence the behaviour of end users, while at the same time keeping them motivated to participate. 
- The cost associated with incentivising a desired behaviour are lower the more intrinsically motivated or the more internalised a goal is for the end user. Therefore, the type of motivation is an important optimisation criterion for the energy supplier or energy services companies (ESCOs) in order to maximise the participation of building occupants, while at the same time minimising costs.

- In our context we see motivation more as a general tendency, while intention is the condition shortly before showing a behaviour in question. Short-term energy optimisation is focused on maximising the behavioural intention to act in order to make sure that the prosumer can be considered a reliable asset in the overall process. However, it is also important to not neglect the long-term motivation of building occupants, as the strength of the motivation amplifies or dampens the intention to act, and also the different types of motivation have different cost implications, as discussed above. Therefore both should be considered important optimisation goals for the energy optimisation as well beyond the short-term impact the optimisation of the intention to act can make.

We presented a behaviour testbed implementation, which brings together various input sources including BMS data, indoor localisation, wireless low-power sensors, and a smartphone application for interacting with the building occupants. The integrated behavioural model is used to bring together all these different systems and rigorously estimate the joint probability distribution of behaviour-relevant parameters using a hidden Markov random field. This enables not only the sending of custom real-time messages to the building occupants, integrating them into the energy optimisation process, but also enables the estimation of the expected impact these messages have on the energy consumption, production, and storage, ultimately allowing this approach to be used as a reliable asset in an aggregated energy optimisation process. Qualitative results are positive, however, larger-scale deployment and longer periods are necessary to derive quantitative results in the long run. Having said that, as the approach constitutes an ongoing social experiment, constantly re-evaluating and learning behaviour relevant parameters, it promises to maintain an accurate representation of the building occupants' motivation. In particular, the short-term predictability of the intention to act, being the probability that an individual will react to a message sent to him/her, makes it useful for a BDR scenario, where the ability to use the energy prosumers as reliable flexibility assets is crucial.

Acknowledgments: This work has received funding from the European Union's Horizon 2020 Research and Innovation programme under Grant agreements no. 696009 and 680517 . The research also has been supported in part by a research grant from Science Foundation Ireland (SFI) and is co-funded under the European Regional Development Fund under Grant Number 13/RC/2077.

Author Contributions: J.B devised the underlying models and integration of behavioural theories. C.B. contributed with the translation into mathematical models. All authors conceived and designed the experiments. J.B. wrote the paper.

Conflicts of Interest: The authors declare no conflict of interest.

\section{References}

1. Crain, P.M. Smart grid test underwhelms. In pilot, few power down to save money. Business \& Management Practices, 30 May 2011.

2. Miller, R.; Golab, L.; Rosenberg, C. Modelling weather effects for impact analysis of residential time-of-use electricity pricing. Energy Policy 2017, 105, 534-546. [CrossRef]

3. Blanke, J.; Beder, C.; Klepal, M. Towards understanding how redefining supply side constrains could shape individual demand side behavior. In Proceedings of the 4th European Conference on Behavior and Energy Efficiency, Coimbra, Portugal, September 2016.

4. Blanke, J. Die Handlungs-Regulationstheorie von Hacker und der Motivationstheoretische Ansatz von LockeELatham; VDM Verlag Dr. Müller: Saarbrücken, Germany, 2008; ISBN 3836433885.

5. Locke, E.; Latham, G. Work Motivation. The high performance cycle. In Work Motivation; Kleinbeck, U., Quast, H.-H., Thierry, H., Häcker, H., Eds.; Psychology Press: London, UK, 1990. 
6. Ajzen, I. Behavioural Intervention Based on the Theory of Planned Behaviour. Available online: https: / www.researchgate.net/publication/245582784_Behavioral_Interventions_Based_on_the_Theory_ of_Planned_Behavior (accessed on 14 December 2017).

7. Bandura, A. Social cognitive theory: An argentic perspective. Asian J. Soc. Psychol. 1999, 2, 21-41. [CrossRef]

8. Self-Determination Theory. Available online: http://selfdeterminationtheory.org/theory/ (accessed on 14 December 2017).

9. Hacker, W. Action regulation theory: A practical tool for the design of modern work processes? Eur. J. Work Organ. Psychol. 2003, 12, 105-130. [CrossRef]

10. Snyder, C.R.; Lopez, S.J. Handbook of Positive Psychology; Oxford University Press: Oxford, UK, 2002.

11. Deci, E.L.; Ryan, R.M. Self-determination theory: A macrotheory of human motivation, development and health. Can. Psychol. 2008, 49, 182-185. [CrossRef]

12. Ryan, R.M.; Deci, E.L. Self-determination theory and the facilitation of intrinsic motivation, social development, and well-being. Am. Psychol. 2000, 55, 68-78. [CrossRef] [PubMed]

13. Osbaldison, R.; Sheldon, K.M. Promoting internalized motivation for environmentally responsible behavior: A prospective study of environmental goals. J. Environ. Psychol. 2003, 23, 349-357. [CrossRef]

14. Pham, L.; Blanke, J. Tackling the value-action-gap: Improving civic participation using a holistic behavioural model approach. In Proceedings of the ICDPCP 2017: 19th International Conference on Democracy, Political and Civic Participation, London, UK, 28-29 June 2017.

15. Burgers, C.; Eden, A.; van Engelenburg, M.D.; Buningh, S. How feedback boosts motivation and play in brain-training game. Comput. Hum. Behav. 2015, 48, 94-103. [CrossRef]

16. Vine, D.; Buys, L.; Morris, P. The effectiveness of energy feedback for conservation and peak demand: A literature review. Open J. Energy Effic. 2013, 2, 7-15. [CrossRef]

17. He, H.A.; Greenberg, S.; Huang, E.M. One size does not fit all: Applying the transtheoretical model to energy feedback technology design. In Proceedings of the SIGCHI Conference on Human Factors in Computing Systems, Atlanta, GA, USA, 10-15 April 2010.

18. Behavioral Demand Response Study_Load Impact Evaluation Report. Available online: http:/ /www.oracle. com/us/industries/utilities/behavioral-demand-response-3628982.pdf (accessed on 14 December 2017).

(C) 2017 by the authors. Licensee MDPI, Basel, Switzerland. This article is an open access article distributed under the terms and conditions of the Creative Commons Attribution (CC BY) license (http://creativecommons.org/licenses/by/4.0/). 